\title{
Protograph-Based LDPC Code Design for Shaped Bit-Metric Decoding
}

\author{
Fabian Steiner, Georg Böcherer and Gianluigi Liva
}

\begin{abstract}
A protograph-based low-density parity-check (LDPC) code design technique for bandwidth-efficient coded modulation is presented. The approach jointly optimizes the LDPC code node degrees and the mapping of the coded bits to the bit-interleaved coded modulation (BICM) bit-channels. For BICM with uniform input and for BICM with probabilistic shaping, binary-input symmetric-output surrogate channels for the code design are used. The constructed codes for uniform inputs perform as good as the multi-edge type codes of Zhang and Kschischang (2013). For 8-ASK and 64-ASK with probabilistic shaping, codes of rates $2 / 3$ and $5 / 6$ with blocklength 64800 are designed, which operate within $0.63 \mathrm{~dB}$ and $0.69 \mathrm{~dB}$ of $\frac{1}{2} \log _{2}(1+\mathbf{S N R})$ for a target frame error rate of $10^{-3}$ at spectral efficiencies of 1.38 and 4.25 bits/channel use, respectively.
\end{abstract}

\section{INTRODUCTION}

Bit-interleaved coded modulation (BICM) combines high order modulation with binary error correcting codes [1], [2]. This makes BICM attractive for practical application and BICM is widely used in standards, e.g., in DVB-T2/S2/C2. At a BICM receiver, bit-metric decoding (BMD) is used [3, Sec. II]. Achievable rates for BMD were investigated for uniformly distributed inputs in [3] and for non-uniformly distributed bits in [4]. These results were generalized to nonuniformly distributed input symbols in [5] and [6]. BICM with probabilistic shaping can achieve rates very close to the capacity $\frac{1}{2} \log _{2}(1+$ SNR $)$ of the additive white Gaussian noise (AWGN) channel [6]. The aim of this work is the design of low-density parity-check (LDPC) codes for BICM with probabilistic shaping to approach $\frac{1}{2} \log _{2}(1+\mathrm{SNR})$ at high spectral efficiencies.

The key aspect of designing LDPC codes for BICM are the unequal error protection of the LDPC coded bits and the BICM bit-channels that are different for different bit-levels. A first approach is to take an existing LDPC code and to optimize the mapping of the coded bits to the BICM bit-levels. This was done, e.g., in [7]-[9]. A more fundamental approach is to directly incorporate the different bit-channels in the code

Fabian Steiner was with the Institute for Communications Engineering, Technische Universität München. He is now with the Institute for Circuit Theory and Signal Processing, Technische Universität München. Georg Böcherer is with the Institute for Communications Engineering, Technische Universität München. Gianluigi Liva is with the Institute of Communication and Navigation of the Deutsches Zentrum für Luft- und Raumfahrt (DLR) in Wessling, Germany. Emails: fabian.steineretum.de, georg.boecherer@tum.de, Gianluigi.Liva@dlr.de

This work was supported by the German Ministry of Education and Research in the framework of an Alexander von Humboldt Professorship.

Parts of this work will be presented at the 2015 IEEE International Symposium on Information Theory (ISIT). design. This is done in [10], where the authors use multiedge type (MET) codes [11] to parameterize the different bitchannels. They then extend the extrinsic information transfer charts (EXIT) [12] to multiple dimensions to design codes for quadrature amplitude modulation with 16 signal points (16-QAM). As 16-QAM can be constructed as the Cartesian product of two four point amplitude-shift keying (4-ASK) constellations, two different bit-channels are apparent. For constellations with more than two different bit-channels, the authors of [10] observe long runtimes of their multidimensional EXIT approach. Therefore, they suggest a high-order extension based on nesting, i.e., starting from $m=2$ bit-levels, they successively extend their codes from $m$ to $m+1$ bit-levels by optimizing in each step only the additional bit-level.

In this work, we follow [10] and jointly optimize the code structure and the mapping of the coded bits to the BICM bit-levels, but propose a protograph-based design [13]. In [14], protographs are designed for coded modulation by a variable degree matched mapping (VDMM). Each bit-level is associated to a specific protograph variable node following the waterfilling approach (i.e., assigning the most protected coded bits to the bit-levels with highest bit-channel capacities). Recently, a protograph-based coded modulation scheme was introduced in [15] by performing a one-to-one mapping between the constellation symbols and the codeword symbols of a non-binary protograph LDPC code. This requires the constellation order to match the field order on which the LDPC code is constructed. To the best of our knowledge, none of the above-mentioned approaches leads to a joint optimization of the binary LDPC protograph ensemble and the bit-mapping.

We propose the following code design. We represent each bit-channel by a biAWGN surrogate. Our surrogates reflect both the BICM bit-channels and the input distribution. For the surrogate channels, we employ protograph EXIT (P-EXIT) analysis [16] to determine the ensemble iterative convergence threshold by accounting for the different bit-channels associated with the protograph variable nodes. The protograph ensemble is optimized with respect to the threshold by differential evolution. We optimize codes both for uniformly distributed inputs and for the probabilistic shaping scheme proposed in [5]. Our optimized codes perform as good as the codes by Zhang and Kschischang [10]. Moreover, our design approach can be applied to arbitrary large constellations with shaped inputs. Our codes of rates $2 / 3$ and $5 / 6$ with blocklength 64800 operate within $0.63 \mathrm{~dB}$ and $0.69 \mathrm{~dB}$ of $\frac{1}{2} \log _{2}(1+\mathrm{SNR})$ for a target frame error rate of $10^{-3}$ at spectral efficiencies of 1.38 and 4.25 bits/channel use, respectively.

This paper is organized as follows. In Sec. II, we review LDPC coded modulation with probabilistic shaping. Sec. III in- 
000001011010110111101100

Figure 1. 8-ASK constellation with BRGC [17] labeling.

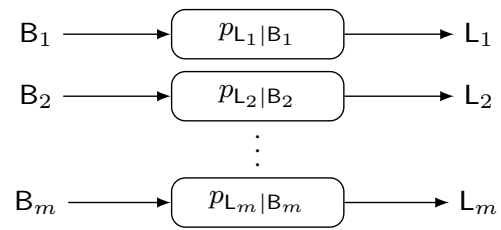

Figure 2. $m$ parallel bit-channels that are different for different bit-levels.

troduces the concept of protographs and explains the necessary adaptions for P-EXIT analysis. We present our code design approach in Sec. IV. In Sec. V, we discuss the performance of our codes for uniform and shaped inputs, respectively. We conclude in Sec. VI.

\section{PRELIMINARIES}

\section{A. Channel Model}

Consider the discrete time AWGN channel

$$
\mathrm{Y}=\Delta \mathrm{X}+\mathrm{Z}
$$

where the noise term $Z$ is zero mean, unit variance Gaussian, and where the input $X$ is distributed on the normalized $2^{m}$ ASK constellation

$$
\mathcal{X}=\left\{ \pm 1, \pm 2, \ldots, \pm\left(2^{m}-1\right)\right\} .
$$

The constellation spacing $\Delta$ controls the average power $\mathrm{E}\left[|\Delta \mathrm{X}|^{2}\right]$, where $\mathrm{E}[\cdot]$ denotes expectation. The signal-to-noise ratio (SNR) is $\mathrm{SNR}=\mathrm{E}\left[|\Delta \mathrm{X}|^{2}\right] / 1$. Each signal point $x \in \mathcal{X}$ is labeled by $m$ bits $\mathbf{B}=\left(\mathrm{B}_{1}, \mathrm{~B}_{2}, \ldots, \mathrm{B}_{m}\right)$, where $\mathrm{B}_{i}$ denotes the $i$-th bit-level. Throughout this work, we label by the binary reflected Gray code (BRGC) [17], e.g., see Fig. 1. Let $p_{\mathrm{Y} \mid \mathrm{B}}$ be the transition function of the memoryless channel with input B and output $Y$. At the receiver, a soft-demapper calculates for each bit-level $i$ the soft-information

$$
L_{i}=\underbrace{\log \frac{p_{\mathrm{Y}_{\mid \mathrm{B}}}(y \mid 0)}{p_{\mathrm{Y}_{\mathrm{B}}}(y \mid 1)}}_{\text {channel likelihood }}+\underbrace{\log \frac{P_{\mathrm{B}_{i}}(0)}{P_{\mathrm{B}_{i}}(1)}}_{\text {input priors }}
$$

which can be calculated by means of the joint distribution

$$
P_{B_{i}}\left(b_{i}\right) p_{\mathrm{Y}_{\mathbf{B}} \mathrm{B}_{i}}\left(y \mid b_{i}\right)=\sum_{\boldsymbol{a} \in\{0,1\}^{m}: a_{i}=b_{i}} p_{\mathbf{Y} \mid \mathbf{B}}(y \mid \boldsymbol{a}) P_{\mathbf{B}}(\boldsymbol{a}) .
$$

A bit-metric decoder uses the soft-information $L_{1}, L_{2}, \ldots, L_{m}$ to estimate the transmitted data. For the decoder, the channel appears as $m$ parallel bit-channels, see Fig. 2. Bit-metric decoding can achieve the rate [6, Theorem 1]

$$
R_{\mathrm{BMD}}=\mathbb{H}(\mathbf{B})-\sum_{i=1}^{m} \mathbb{H}\left(\mathrm{B}_{i} \mid \mathrm{Y}\right)=\mathbb{H}(\mathbf{B})-\sum_{i=1}^{m} \mathbb{H}\left(\mathrm{B}_{i} \mid \mathrm{L}_{i}\right)
$$

where $\mathbb{H}(\cdot)$ denotes entropy.

Remark 1. If the bits $\mathrm{B}_{1}, \mathrm{~B}_{2}, \ldots, \mathrm{B}_{m}$ are independent, then (5) can be written as [3], [4]

$$
R_{\mathrm{BMD}}=\sum_{i=1}^{m} \mathrm{I}\left(\mathrm{B}_{i} ; \mathrm{L}_{i}\right)
$$

where $\mathbb{I}(\cdot ; \cdot)$ denotes mutual information.

\section{B. LDPC Coded Modulation}

1) LDPC codes: LDPC codes are linear block codes with a sparse $(n-k) \times n$ parity-check matrix $\boldsymbol{H}$. The matrix $\boldsymbol{H}$ can be represented by a Tanner graph [18, Section 3.3] consisting of variable nodes $V_{i}, i \in\{1, \ldots, n\}$ and check nodes $C_{j}, j \in$ $\{1, \ldots, n-k\}$. In the following, the set $\mathcal{N}\left(V_{i}\right)$ denotes all neighbors of variable node $V_{i}$, i.e., all check nodes $C_{j}$ with a one occuring in the $i$-th column of $\boldsymbol{H}$; the set $\mathcal{N}\left(C_{j}\right)$ is defined as all variable nodes $V_{i}$ possessing a one in the $j$-th row of $\boldsymbol{H}$. The variable and check node degrees of the $i$-th variable and $j$-th check node are then given as the cardinality of the sets $\mathcal{N}\left(V_{i}\right)$ and $\mathcal{N}\left(C_{j}\right)$, respectively.

2) Transmitter: The canonical model of a BICM transmitter [19, Sec. 2.3] is displayed in Fig. 3a. $k$ bits are encoded by a rate $k / n$ encoder. The coded bits are then permuted by an interleaver and a mapper modulates the bits to a sequence of channel input symbols of length $n_{\mathrm{c}}=n / m$. We adapt this general BICM model as follows. We describe the mapping function by specifying to which bit-level each coded bit is mapped. The mapper input is

$$
\left(\mathrm{B}_{2} \cdots \mathrm{B}_{m}\right)^{n_{\mathrm{c}}} \mathrm{B}_{1}^{n_{\mathrm{c}}}
$$

where

$$
\left(\mathrm{B}_{2} \cdots \mathrm{B}_{m}\right)^{\eta_{\mathrm{c}}}=\left(\mathrm{B}_{2} \cdots \mathrm{B}_{m}\right)_{1}\left(\mathrm{~B}_{2} \cdots \mathrm{B}_{m}\right)_{2} \cdots\left(\mathrm{B}_{2} \cdots \mathrm{B}_{m}\right)_{n_{\mathrm{c}}}
$$
and

$$
\mathrm{B}_{1}^{n_{\mathrm{c}}}=\mathrm{B}_{11} \mathrm{~B}_{12} \cdots \mathrm{B}_{1 n_{\mathrm{c}}} \text {. }
$$

We put the bits for bit-level 1 in the end. At this point, this choice is arbitrary, but it will become useful later on when we consider shaped inputs. The memoryless modulation function of the mapper is

$$
B_{1 i} B_{2_{i}} \cdots B_{m i} \mapsto x_{B_{1 i} B_{2 i} \cdots B_{m i}} .
$$

For LDPC coded BICM, each coded bit has a variable node degree and it is transmitted over one of $m$ bit-channels. The resulting mapping of variable node degrees to bit-levels strongly influences the error performance of the system [7]. If the LDPC code is given and fixed, we have to choose the interleaver appropriately. Since we are going to design new LDPC codes, we instead remove the interleaver and account for the mapping of variable node degrees to bit-levels in our code design. We choose a systematic rate $(m-1) / m$ LDPC encoder, which realizes the mapping

$$
\left(\mathrm{B}_{2} \cdots \mathrm{B}_{m}\right)^{n_{\mathrm{c}}} \mapsto\left(\mathrm{B}_{2} \cdots \mathrm{B}_{m}\right)^{n_{\mathrm{c}}} \mathrm{B}_{1}^{n_{\mathrm{c}}} .
$$

Our model can be extended to code rates higher than $(m-$ $1) / m$, see [21]. For clarity of exposure, we only consider rate $(m-1) / m$ codes in the present work. 


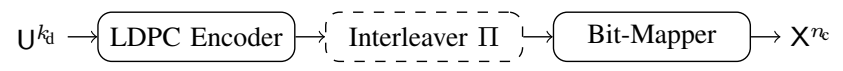

(a) Canonical BICM model [19, Sec. 2.3].

$\mathrm{U}^{k_{\mathrm{d}}} \rightarrow$ Matcher $\rightarrow\left(\mathrm{B}_{2} \cdots \mathrm{B}_{m}\right)^{n_{\mathrm{c}}} \rightarrow$ LDPC Encoder $\rightarrow\left(\mathrm{B}_{2} \cdots \mathrm{B}_{m}\right)^{n_{\mathrm{c}}} \mathrm{B}_{1}^{n_{\mathrm{c}}} \rightarrow$ Bit-Mapper $\rightarrow \mathrm{X}^{n_{\mathrm{c}}}$

(b) BICM with probabilistic shaping [5].

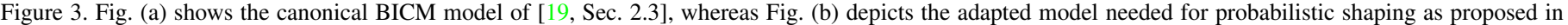

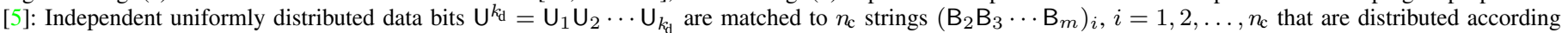

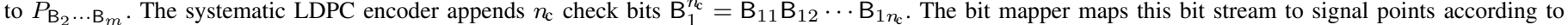

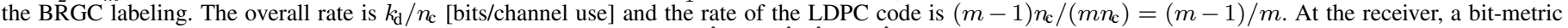

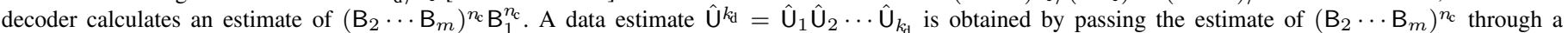
dematcher. For the matcher and the dematcher, we use [20].

3) Shaped Inputs: We use the shaping scheme proposed in [21]. The capacity-achieving distribution of ASK constellations in Gaussian noise is symmetric around the origin. Consequently, it induces a distribution $P_{\mathrm{B}_{1} \mathrm{~B}_{2} \cdots \mathrm{B}_{m}}$ on the BRGC labeling with the following properties:

- Bit-level $B_{1}$ decides on the sign of the transmitted constellation point, see Fig. 1. Because of symmetry, bit-level $B_{1}$ is uniformly distributed.

- Bit-levels $\left(B_{2} \cdots B_{m}\right)$ and bit-level $B_{1}$ are independent. Bit-levels $B_{2}, \ldots, B_{m}$ are correlated.

The scheme [21] mimics the capacity-achieving distribution in the following way: first, generate bit-levels $\mathrm{B}_{2} \cdots \mathrm{B}_{m}$ according to $P_{\mathrm{B}_{2} \cdots \mathrm{B}_{m}}$, e.g., by using a distribution matcher (see Fig. 3b). In our implementation, we use the distribution matcher proposed in [20]. The systematic encoder copies the bits $\left(\mathrm{B}_{2} \cdots \mathrm{B}_{m}\right)^{n_{\mathrm{c}}}$ to its output and leaves their distribution un-changed. The encoder appends check bits $B_{1}^{n_{c}}$ that are approximately uniformly distributed because each check bit is a modulo two sum of many information bits [22, Sec. 7.1]. The signal point $x_{\mathrm{B}_{1} \mathrm{~B}_{2} \cdots \mathrm{B}_{m}}$ selected by the bit-mapper then has approximately the capacity-achieving distribution.

\section{PROTOGRAPHS AND EXIT ANALYSIS FOR PARALLEL CHANNELS}

\section{A. Protograph-Based LDPC codes}

LDPC code ensembles as introduced in II-B1 are usually characterized by the degree profiles of the variable and check nodes. For instance, $\lambda(x)=\sum_{d=1}^{d_{v}} \lambda_{d} x^{d-1}$ and $\rho(x)=$ $\sum_{d=1}^{d_{c}} \rho_{d} x^{d-1}$ are the edge-perspective variable and check node degree polynomials with maximum degree $d_{v}$ and $d_{c}$, respectively. However, the degree profiles do not allow to characterize the mapping of variable nodes to the $m$ different bit-channels resulting from our adapted BICM transmission scheme. In the following, we use protographs to incorporate the bit-mapping in our threshold analysis.

Parity-check matrices are constructed from protographs as follows. Starting from a small bipartite graph represented via its basematrix $\boldsymbol{A}=\left[a_{l k}\right]$ of size $M \times N$, one applies a copyand-permute operation (also known as lifting) to create $Q$ instances of the small graph and then permutes the edges so that the local edge connectivity remains the same. The $Q$ replicas of variable node $V_{k}, k \in\{1, \ldots, N\}$ must be connected only to replicas of the neighbors of $V_{k}$ while maintaining the

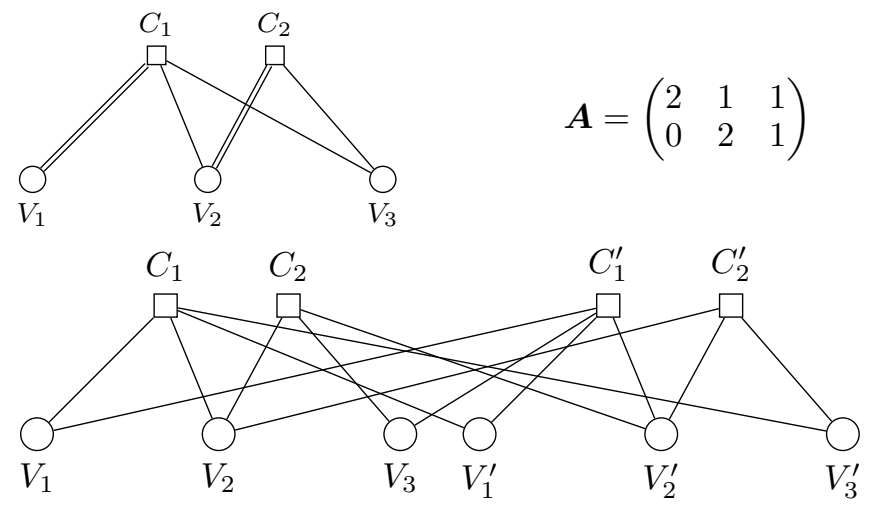

Figure 4. Above, the Tanner graph of basematrix $\boldsymbol{A}$ with $M=2$ and $N=3$ is displayed. Below, an example lifting with $Q=2$ instances of the protograph is shown.

original degrees for that specific edge. The resulting bipartite graph representing the final parity-check matrix $\boldsymbol{H}$ possesses $n=Q \cdot N$ variable nodes and $n-k=Q \cdot M$ check nodes. Parallel edges are allowed, but must be resolved during the copy-and-permute procedure. An example protograph with the corresponding basematrix and an example lifting for $Q=2$ are shown in Figure 4.

\section{B. Protograph EXIT}

The performance of protograph-based LDPC codes over parallel channels can be easily analyzed in the infinite blocklength limit by suitably modifying the P-EXIT. We consider next the case where the codeword bits corresponding to the $N$ protograph variable nodes are transmitted over $N$ binaryinput symmetric-output parallel channels. In the following, we denote by $\mathrm{I}_{V_{k} \rightarrow C_{l}, \ell}^{\mathrm{E}}$ the mutual information (MI) between the message sent at iteration $\ell$ by the $k$-th variable node to the $l$-th check node and the corresponding codeword bit. Similarly, $\mathrm{I}_{C_{l} \rightarrow V_{k}}^{\mathrm{E}, \ell}$ denotes the MI between the message sent at iteration $\ell$ by the $l$-th check node to the $k$-th variable node and the corresponding codeword bit. We further express the MI between the $k$-th channel output and input as $\mathrm{I}_{k}^{\mathrm{ch}}$. The evolution 


$$
\begin{aligned}
& \mathrm{I}_{V_{k} \rightarrow C_{l}}^{\mathrm{E}, \ell}=\mathrm{J}\left(\sqrt{\sum_{\substack{l^{\prime}=1 \\
l^{\prime} \neq l}}^{M} a_{l^{\prime} k} \cdot \mathrm{J}^{-1}\left(\mathrm{I}_{C_{l^{\prime}} \rightarrow V_{k}}^{\mathrm{E}, \ell-1}\right)^{2}+\left(a_{l k}-1\right) \cdot \mathrm{J}^{-1}\left(\mathrm{I}_{C_{l} \rightarrow V_{k}}^{\mathrm{E}, \ell-1}\right)^{2}+\sigma_{\mathrm{ch}, k}^{2}}\right) \\
& \mathrm{I}_{C_{l} \rightarrow V_{k}}^{\mathrm{E}, \ell}=1-\mathrm{J}\left(\sqrt{\sum_{\substack{k^{\prime}=1 \\
k^{\prime} \neq k}}^{N} a_{l k^{\prime}} \mathrm{J}^{-1}\left(1-\mathrm{I}_{V_{k^{\prime}} \rightarrow C_{l}}^{\mathrm{E}, \ell}\right)^{2}+\left(a_{l k}-1\right) \cdot \mathrm{J}^{-1}\left(1-\mathrm{I}_{V_{k} \rightarrow C_{l}}^{\mathrm{E}, \ell}\right)^{2}}\right) \\
& \mathrm{I}_{k}^{\mathrm{APP}, \ell}=\mathrm{J}\left(\sqrt{\sum_{l^{\prime}=1}^{M} a_{l^{\prime} k} \cdot \mathrm{J}^{-1}\left(\mathrm{I}_{C_{l^{\prime}} \rightarrow V_{k}}^{\mathrm{E}, \ell}\right)^{2}+\sigma_{\mathrm{ch}, k}^{2}}\right) .
\end{aligned}
$$

of the MI can be tracked by applying the recursion

$$
\begin{aligned}
& \mathrm{I}_{V_{k} \rightarrow C_{l}}^{\mathrm{E}, \ell}=\mathrm{f}_{k, l}^{\mathrm{V}}\left(\mathbf{I}_{C \rightarrow V_{k}}^{\mathrm{E}, \ell-1}, \mathrm{I}_{k}^{\mathrm{ch}}\right) \\
& \mathrm{I}_{C_{l} \rightarrow V_{k}}^{\mathrm{E}, \ell}=\mathrm{f}_{k, l}^{\mathrm{C}}\left(\mathbf{I}_{V \rightarrow C_{l}}^{\mathrm{E}, \ell}\right)
\end{aligned}
$$

with

$$
\mathbf{I}_{C \rightarrow V_{k}}^{\mathrm{E}, \ell}:=\left(\mathrm{I}_{C_{1} \rightarrow V_{k}}^{\mathrm{E}, \ell}, \mathrm{I}_{C_{2} \rightarrow V_{k}}^{\mathrm{E}, \ell}, \ldots, \mathrm{I}_{C_{M} \rightarrow V_{k}}^{\mathrm{E}, \ell}\right)
$$

and

$$
\mathbf{I}_{V \rightarrow C_{l}}^{\mathrm{E}, \ell}:=\left(\mathrm{I}_{V_{1} \rightarrow C_{l}}^{\mathrm{E}, \ell}, \mathrm{I}_{V_{2} \rightarrow C_{l}}^{\mathrm{E}, \ell}, \ldots, \mathrm{I}_{V_{N} \rightarrow C_{l}}^{\mathrm{E}, \ell}\right)
$$

where by convention we set $\mathrm{I}_{V_{k} \rightarrow C_{l}}^{\mathrm{E}, \ell}=\mathrm{I}_{C_{l} \rightarrow V_{k}}^{\mathrm{E}, \ell}=0$ if $C_{l} \notin \mathcal{N}\left(V_{k}\right)$. In (12), (13) we introduced the variable and check extrinsic information transfer (EXIT) functions $\mathrm{f}_{k, l}^{\mathrm{V}}$ and $\mathrm{f}_{k, l}^{C}$, whose form depends on the underlying channel model. We moreover denote by $\mathrm{I}_{k}^{\mathrm{APP}, \ell}$ the MI between the a posteriori probability (APP) log-likelihood ratio (LLR) message computed at the $k$-th variable node in the $\ell$-th iteration, and the corresponding codeword bit. Note that $\mathrm{I}_{k}^{\mathrm{APP}, \ell}$ is a function of $\mathbf{I}_{C \rightarrow V_{k}}^{\mathrm{E}, \ell-1}$ and $\mathbf{I}_{k}^{\mathrm{ch}}$.

Following [23], we define the protograph convergence region $C_{\mathrm{I}}$ as the set of channel MI vectors $\mathbf{I}^{\mathrm{ch}}=\left(\mathrm{I}_{1}^{\mathrm{ch}}, \mathrm{I}_{2}^{\mathrm{ch}}, \ldots, \mathrm{I}_{N}^{\mathrm{ch}}\right)$ for which $\mathrm{I}_{k}^{\mathrm{APP}, \ell}$ converges to 1 for all $k \in[1, N]$, as $\ell \rightarrow \infty$,

$$
C_{\mathbf{l}^{\mathrm{ch}}}:=\left\{\mathbf{I}^{\mathrm{ch}} \mid \mathrm{I}_{k}^{\mathrm{APP}, \ell} \rightarrow 1, \forall k, \ell \rightarrow \infty\right\} .
$$

We will sometimes restate the convergence region with respect to other parameters characterizing the channel nuisance. Hence, we will denote the convergence region with respect to the generic channel parameter $\boldsymbol{\xi}$ as $C_{\boldsymbol{\xi}}$.

Example 1 (Convergence region of parallel binary-input additive white Gaussian noise channels). The convergence region of $N$ parallel binary input additive white Gaussian noise (biAWGN) channels, with noise variances $\sigma_{\mathrm{ch}, k}^{2}$ and inputs alphabets $\left\{ \pm \sigma_{\mathrm{ch}, k}^{2} / 2\right\}, k=1, \ldots, N$, may be expressed with respect to the noise standard deviation as

$$
C_{\boldsymbol{\sigma}_{\mathrm{ch}}}:=\left\{\sigma_{\mathrm{ch}, 1}, \sigma_{\mathrm{ch}, 2}, \ldots, \sigma_{\mathrm{ch}, N} \mid \mathrm{I}_{k}^{\mathrm{APP}, \ell} \rightarrow 1, \forall k, \ell \rightarrow \infty\right\} .
$$

The evaluation of (12) and (13) at the $\ell$-th iteration is given by (9) and (10), with $\mathrm{I}_{k}^{\mathrm{APP}, \ell}$ evaluated as in (11) and where $\mathrm{J}\left(\sigma_{\mathrm{ch}}\right)$ is

$$
\mathrm{J}\left(\sigma_{\mathrm{ch}}\right):=1-\int_{-\infty}^{\infty} \frac{1}{\sqrt{2 \pi \sigma_{\mathrm{ch}}^{2}}} \mathrm{e}^{-\frac{\left(z-\sigma_{\mathrm{ch}}^{2} / 2\right)^{2}}{2 \sigma_{\mathrm{ch}}^{2}}} \log _{2}\left(1+\mathrm{e}^{-z}\right) \mathrm{d} z
$$

Example 2 (Convergence region of parallel binary erasure channels). If the underlying parallel channels are binary erasure channels (BECs) with erasure probabilities $\epsilon_{k}, k=$ $1, \ldots, N$, the convergence region in terms of erasure probability is denoted by

$$
C_{\epsilon}:=\left\{\epsilon_{1}, \epsilon_{2}, \ldots, \epsilon_{N} \mid \mathrm{I}_{k}^{\mathrm{APP}, \ell} \rightarrow 1, \forall k, \ell \rightarrow \infty\right\} .
$$

Here, the recursion (12), (13) at the $\ell$-th iteration is given by

$$
\left.\begin{array}{l}
\mathrm{I}_{V_{k} \rightarrow C_{l}}^{\mathrm{E}, \ell}=1-\epsilon_{k}\left(1-\mathrm{I}_{C_{l} \rightarrow V_{k}}^{\mathrm{E}, \ell-1}\right)^{a_{l k}-1} \prod_{\substack{l^{\prime}=1 \\
l^{\prime} \neq l}}^{M}\left(1-\mathrm{I}_{C_{l^{\prime}} \rightarrow V_{k}}^{\mathrm{E}, \ell-1}\right)^{a_{l^{\prime} k}} \\
\mathrm{I}_{C_{l} \rightarrow V_{k}}^{\mathrm{E}, \ell}=\left(\mathrm{I}_{V_{k} \rightarrow C_{l}}^{\mathrm{E}, \ell}\right)^{a_{l k}-1} \prod_{\substack{k^{\prime}=1 \\
k^{\prime} \neq k}}^{N}\left(\mathrm{I}_{V_{k^{\prime}}, \ell}^{\mathrm{E}, \ell} C_{l}\right.
\end{array}\right)^{a_{l k^{\prime}}}
$$

with

$$
\mathrm{I}_{k}^{\mathrm{APP}, \ell}=1-\epsilon_{k} \prod_{l^{\prime}=1}^{M}\left(1-\mathrm{I}_{C_{l^{\prime}} \rightarrow V_{k}}^{\mathrm{E}, \ell-1}\right)^{a_{l^{\prime} k}}
$$

\section{Code Design via Surrogate Channels}

The $m$ bit-channels in Fig. 2 are not output-symmetric and the input is not uniformly distributed when we perform shaping. Optimizing LDPC codes directly for the original bitchannels in Fig. 2 is therefore difficult. To overcome this problem, we use the paradigm of universal codes. A code is universal, if it performs similar on different channels, under certain conditions. Universality allows to replace the original channel by a surrogate channel for which code design is easier. We first derive a universality criterion and then construct surrogate channels. 


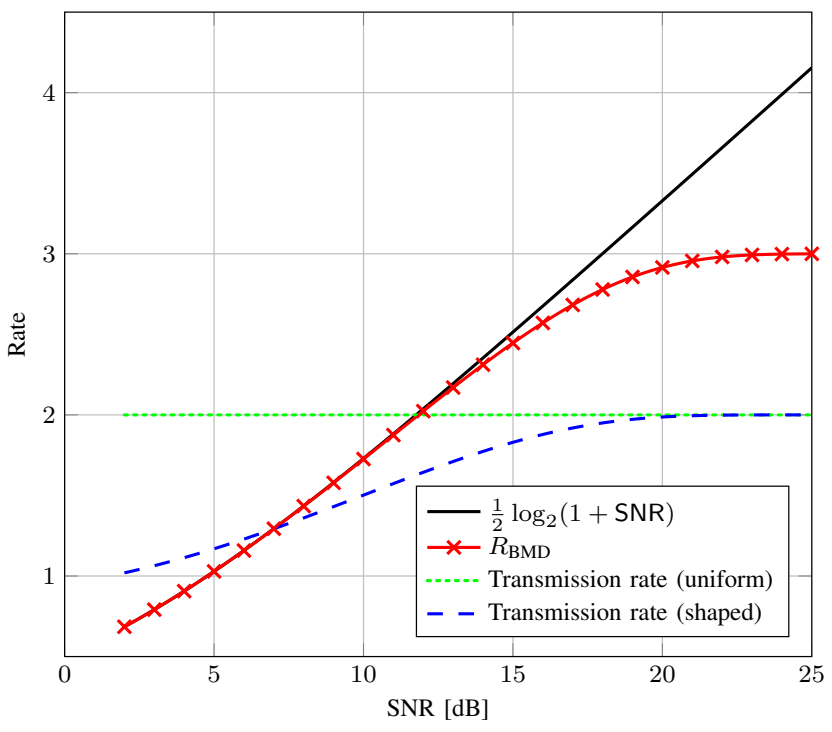

Figure 5. Search trajectory for protograph optimization for uniform and shaped inputs.

\section{A. A Universality Criterion}

We start by considering the rate backoff criterion [21, Sec. VIII.C]. Let $R^{*}$ be the rate that can asymptotically be achieved by the considered code ensemble on the original channel and let $R$ be the actual transmission rate. The rate backoff is

$$
R^{*}-R \text {. }
$$

In [21, Sec. VIII.C], it is assumed that the error performance is a function of $R^{*}-R$. Consider our transmission scheme of Fig. 3. Suppose the code rate is $c$ so that $(1-c) m$ bits per channel use consist of uniformly distributed redundancy bits on average and suppose further that the input distribution is $P_{\mathrm{B}}$. Then, by (5) and Sec. II-B, the achievable rate and the transmission rate, respectively, are given by

$$
\begin{aligned}
& R^{*}=\mathbb{H}(\mathbf{B})-\sum_{i=1}^{m} \mathbb{H}\left(\mathrm{B}_{i} \mid \mathrm{L}_{i}\right) \\
& R=\mathbb{H}(\mathbf{B})-(1-c) m
\end{aligned}
$$

Taking the difference, we have

$$
R^{*}-R=(1-c) m-\sum_{i=1}^{m} \mathbb{H}\left(\mathrm{B}_{i} \mid \mathrm{L}_{i}\right) .
$$

Note that the term $(1-c) m$ does not depend on the statistics, so that the sum of the conditional entropies determines the rate backoff. Since we want to account for the different bitchannels in our code design, we strengthen the rate backoff criterion and use as criterion the bit uncertainties

$$
\mathcal{U}=\left\{\mathbb{H}\left(\mathrm{B}_{i} \mid \mathrm{L}_{i}\right)\right\}_{i=1}^{m} .
$$

\section{B. Surrogate Channels}

Suppose we optimized a code on some surrogate channel. If the performance of the code on the original channel cannot be improved by changing the bit-mapping, we succeeded to jointly optimize the code and the bit-mapping. In this case, we say that the surrogate channel is proper. If in contrary the performance of the optimized code on the original channel can be improved by changing the bit-mapping, our joint optimization attempt failed. We will use the following two surrogate channels for code optimization.

1) BEC Surrogate: We replace the original transmission scheme by a scheme where all input bits $\tilde{B}_{i}$ are uniformly distributed and where each bit-channel $P_{\tilde{L}_{i} \mid \tilde{B}_{i}}$ is a binary erasure channel (BEC) with erasure probability $\epsilon_{i}$. Criterion (18) requires

$$
\mathbb{H}\left(\tilde{\mathrm{B}}_{i} \mid \tilde{\mathrm{L}}_{i}\right)=\epsilon_{i} \stackrel{!}{=} \mathbb{H}\left(\mathrm{B}_{i} \mid \mathrm{L}_{i}\right), \quad i=1,2, \ldots, m .
$$

2) biAWGN Surrogate: All input bits $\tilde{B}_{i}$ are uniformly distributed and transmitted over biAWGN channels

$$
\tilde{\mathrm{L}}_{i}=x_{\tilde{\mathrm{B}}_{i}}+\mathrm{Z}_{i}
$$

where $x_{\tilde{\mathrm{B}}_{i}} \in\left\{ \pm \sigma_{\mathrm{ch}, i}^{2} / 2\right\}$ and the noise $\mathrm{Z}_{i}$ has variance $\sigma_{\mathrm{ch}, i}^{2}$. Applying criterion (18) yields

$$
\mathbb{H}\left(\tilde{\mathrm{B}}_{i} \mid \tilde{\mathrm{L}}_{i}\right) \stackrel{!}{=} \mathbb{H}\left(\mathrm{B}_{i} \mid \mathrm{L}_{i}\right), \quad i=1,2, \ldots, m .
$$

This can be achieved by numerically solving for $\sigma_{\mathrm{ch}, i}$.

\section{Iterative Decoding Threshhold}

Since irregular LDPC codes have shown better performance than regular LDPC codes [24], we want to allow different variable node degrees for each of the $m$ distinct bit-channels as well. In order to have up to $D$ different degrees per bitchannel, the protograph matrix $\boldsymbol{A}$ must have at least $N=D \cdot m$ variable nodes. We introduce a mapping function of the form $T(k)=\lceil k / D\rceil$ to relate each variable node $V_{k}$ with a corresponding bit-level $T(k) \in\{1, \ldots, m\}$. We next define the iterative decoding threshholds for uniform and shaped inputs, respectively.

1) Uniform Inputs: For uniform inputs, the bit uncertainty set

$$
\mathcal{U}=\left\{\mathbb{H}\left(\mathrm{B}_{i} \mid \mathrm{L}_{i}\right)\right\}_{i=1}^{m}=\left\{\mathbb{H}\left(\mathrm{B}_{i} \mid \Delta x_{\mathbf{B}}+Z\right)\right\}_{i=1}
$$

is a function of the SNR via the constellation scaling $\Delta$. Let $\xi^{\mathcal{U}(\mathrm{SNR})}$ denote the vector of surrogate channel parameters associated with the uncertainty set $\mathcal{U}(\mathrm{SNR})$. Given a protograph ensemble, its iterative decoding threshold is given by

$$
\min \text { SNR s.t. } \boldsymbol{\xi}^{\mathcal{U}(\mathrm{SNR})} \in C_{\boldsymbol{\xi}} \text {. }
$$

Thus, differential evolution [25] can then be employed to find the protograph ensemble with the lowest threshold. We next instantiate the iterative decoding threshhold for BEC surrogates and biAWGN surrogates.

Definition 1 (Iterative decoding threshold under BEC surrogate). The iterative decoding threshold of a protograph under 


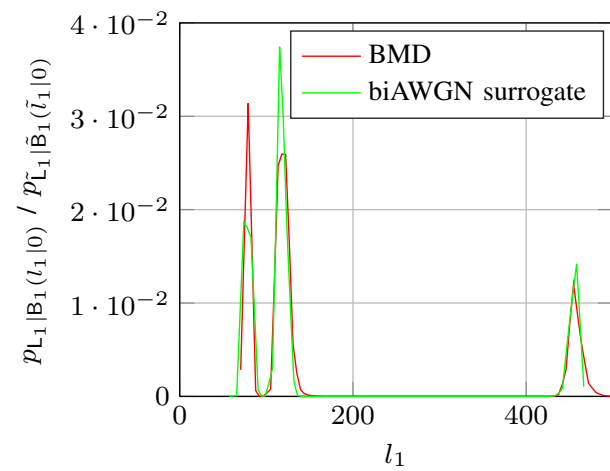

(a) Bit-level $B_{1}$.

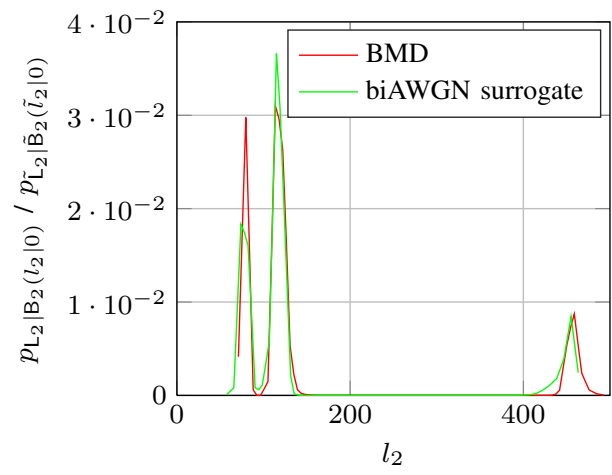

(b) Bit-level $B_{2}$.

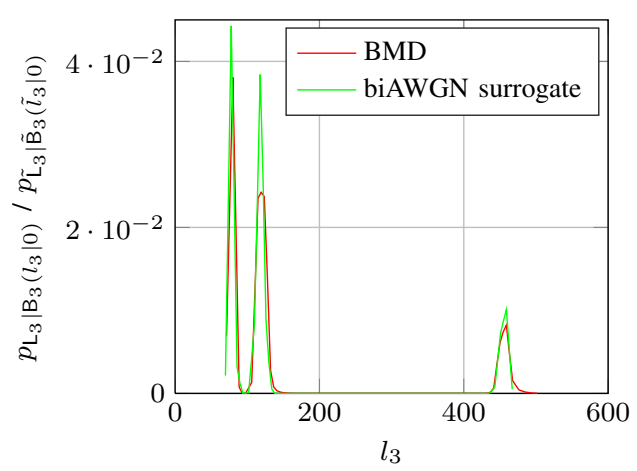

(c) Bit-level $B_{3}$.

Figure 6. Densities of $L$-values for 8-ASK with shaped inputs after 100 iterations at $\mathrm{SNR}=8.1 \mathrm{~dB}$.

the BEC surrogate is the minimum SNR on the original channel such that the resulting erasure probability vector $\varepsilon=\left(\epsilon_{T(1)}, \epsilon_{T(2)}, \ldots, \epsilon_{T(N)}\right)$ of the BEC surrogates belongs to the protograph convergence region $C_{\varepsilon}$.

Definition 2 (Iterative decoding threshold under the biAWGN surrogate). The iterative decoding threshold of a protograph under biAWGN surrogate is the minimum SNR on the original channel such that the resulting LLR standard deviation vector $\boldsymbol{\sigma}_{\mathrm{ch}}=\left(\sigma_{\mathrm{ch}, T(1)}, \sigma_{\mathrm{ch}, T(2)}, \ldots, \sigma_{\mathrm{ch}, T(N)}\right)$ of the biAWGN surrogates belongs to the protograph convergence region $C_{\sigma_{\mathrm{ch}}}$.

2) Shaped Input: For shaped input, the definition of the iterative decoding threshold is ambiguous. Since both the input distribution $P_{\mathrm{B}}$ and the constellation scaling $\Delta$ influence both SNR and the bit-uncertainty set, there are infinitely many bituncertainty sets corresponding to the same value of SNR. We use an information theoretic argument to uniquely parametrize the bit-uncertainty set as a function of SNR. For each SNR, we choose $P_{\mathrm{B}}$ and $\Delta$ that maximize the achievable rate, i.e., we let $P_{\mathbf{B}}(\mathrm{SNR})$ and $\Delta(\mathrm{SNR})$ be the solutions of

$$
\max _{\Delta, P_{\mathbf{B}}: \mathrm{E}\left[\left|\Delta x_{\mathbf{B}}\right|^{2}\right] \leq \mathrm{SNR}}\left\{\mathbb{H}(\mathbf{B})-\sum_{i=1}^{m} \mathbb{H}\left(\mathbf{B}_{i} \mid x_{\mathbf{B}}+\mathbf{Z}\right)\right\} .
$$

As in the uniform case, the bit uncertainty set is now parametrized by the SNR and we define the threshhold by (23). Definitions 1 and 2 now apply accordingly for shaped input. Note that for shaped input, we do not search along a constant rate but we search along the transmission rate. In Fig. 5, we display example search trajectories for uniform inputs and for shaped inputs for 8-ASK: In case of uniform inputs, the transmission rate is 2 bits/channel use for a rate $(m-1) / m$ code independently of the SNR (dotted curve), whereas the transmission rate is given by $\mathbb{H}(\mathbf{B})-1$ in case of shaped input (dashed curve). In the following, we must keep in mind that for shaped input, changing the SNR also changes the transmission rate.

\section{Simulation Results of the Optimized Codes}

We choose the basematrix $\boldsymbol{A}$ from the set $\{0,1, \ldots, S\}^{M \times N}$. The parameters $M$ and $N$ are chosen such that the desired code rate $c=(N-M) / N$ is met and the number of different degrees per bit-level $D$ (see IV-B) is guaranteed. The maximum number of parallel edges $S$ is crucial for both the performance of the code and the success of the optimization procedure. The product $M \cdot S$ describes the maximum variable node degree in the final code and thereby determines the size of the optimization search space. The optimization of the basematrices is performed as described in IV-C.

\section{A. Properness of BEC and biAWGN Surrogates}

We designed codes using both BEC and biAWGN surrogates. Our simulations results reveal that BEC surrogates are not proper, i.e., the performance of the obtained codes on the original channel can be improved further by changing the bitmapper. For the codes we obtained by using biAWGN surrogates, the resulting bit-mapper are optimal, i.e., changing the bit-mapping degrades the performance on the original channel. Thus, for the considered scenario, the biAWGN channel is a proper surrogate and can be used for code design.

This observation is also verified by comparing the densities of the $L$-values of both the original bit-channels and the biAWGN surrogates after decoding as illustrated in Fig. 6. The plot shows the densities $p_{\mathrm{L}_{i} \mid \mathrm{B}_{i}}\left(l_{i} \mid 0\right)$ and $p_{\tilde{\mathrm{L}}_{i} \mid \tilde{\mathrm{B}}_{i}}\left(\tilde{l}_{i} \mid 0\right)$ (see IV-B2) for 8-ASK with shaped input at SNR $=8.1 \mathrm{~dB}$. We observe a close match of the two densities.

To further illustrate the close matching of the biAWGN surrogates with the original channel, we also plot simulation results for the biAWGN surrogates, see the curves with triangle markers in Fig. 9, Fig. 10 For each SNR, we calculate the corresponding set of surrogate channel parameters $\sigma_{\mathrm{ch}}^{\mathcal{U}(\mathrm{SNR})}$ and simulate transmission over the corresponding uniform input symmetric-output biAWGN channels.

\section{B. Code Design for Uniform Input}

In order to compare our code design approach to the setting of Zhang and Kschischang [10], we design codes of rates $1 / 2$ and $3 / 4$ for 4-ASK constellations with uniform inputs. The optimized protographs are depicted in Table I. 
Table I. OPTIMIZED PROTOGRAPHS AND DESIGN PARAMETERS FOR UNIFORM INPUT.

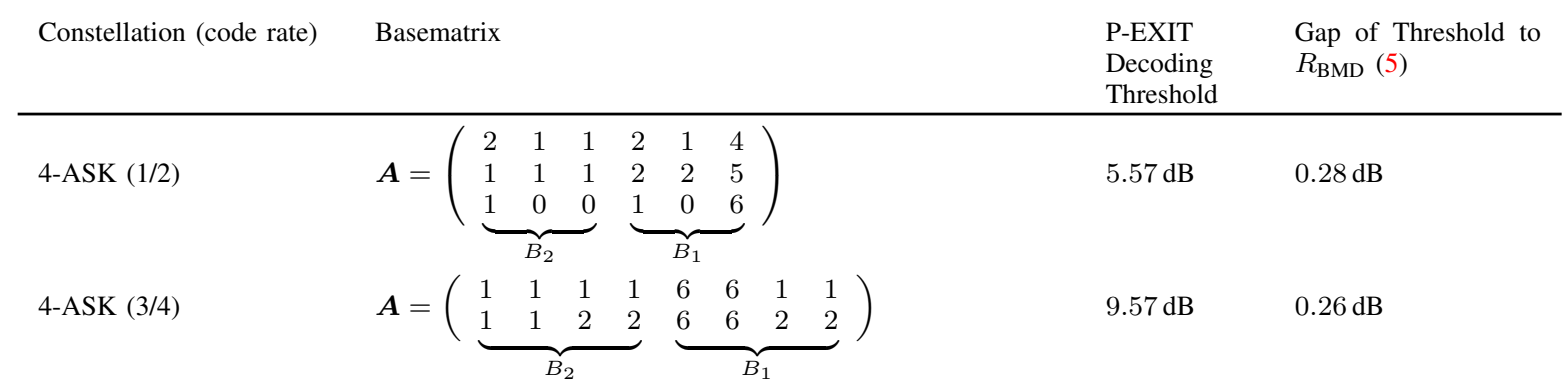

Table II. OPTIMIZED PROTOGRAPHS AND DESIGN PARAMETERS FOR SHAPED INPUT.

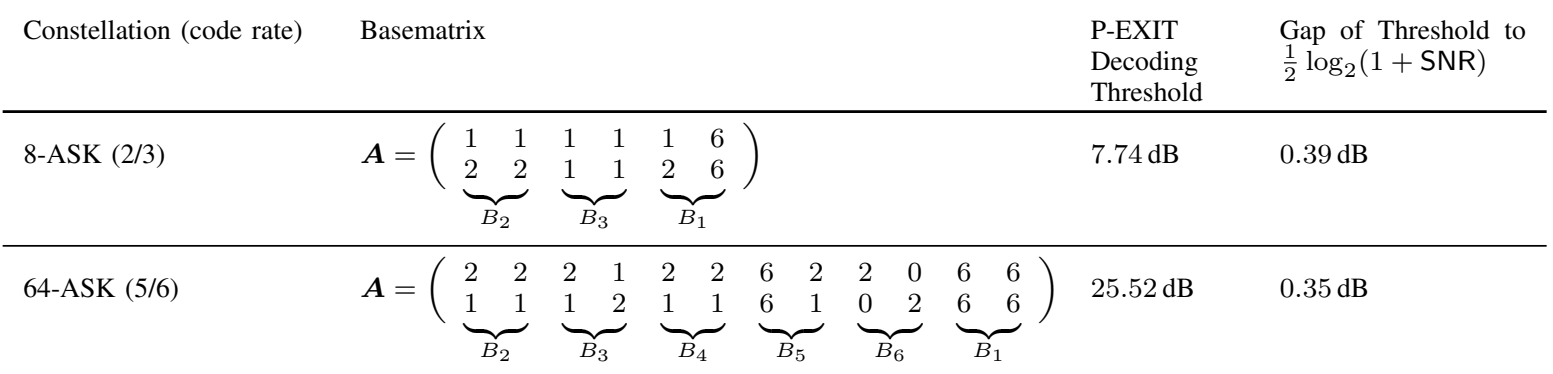

We discuss the rate $1 / 2$ code in more detail. The coded bits are transmitted over two different bit-channels. For each bitchannel, we allow $D=3$ (possibly different) variable degrees so that always 3 variable nodes in the protograph are assigned to the same surrogate channel: $\sigma_{\mathrm{ch}, 1}=\sigma_{\mathrm{ch}, 2}=\sigma_{\mathrm{ch}, 3}$ and $\sigma_{\mathrm{ch}, 4}=\sigma_{\mathrm{ch}, 5}=\sigma_{\mathrm{ch}, 6}$. Once the optimized basematrices have been found, we construct quasi-cyclic parity-check matrices with blocklengths $n=16200$. We simulate the constructed codes using 100 decoding iterations. The bit error rates (BER) and frame error rates (FER) in Fig. 7 and 8 show that the finite length performance of our codes is equal to or slightly better than the codes in [10]. As a reference, we also plot the Shannon limit for BMD and a 4-ASK constellation for the corresponding code rates $1 / 2$ and $3 / 4$. The decoding thresholds given in Table I are obtained by PEXIT analysis for the surrogate channels.

\section{Code Design for Shaped Input}

In the following we design codes of rate $2 / 3$ for 8 -ASK and rate $5 / 6$ for 64 -ASK to show that our design technique scales with the number of bit-levels. The optimized base matrices can be found in Table II. In order to limit the search space for the optimization, we choose $D=2$ and allow a maximum of $S=6$ parallel edges. For comparison, we use the off-theshelf DVB-S2 LDPC codes used in [21] and use the optimized bit-mappers for DVB-S2 codes listed in [21, Table V].

As the shaped input causes a non-constant transmission rate as shown in IV-C2, the bit and frame error ratios are plotted over the gap to continuous AWGN capacity, i.e.,

$$
\left.\Delta \mathrm{SNR}\right|_{\mathrm{dB}}=\left.\operatorname{SNR}(\mathrm{FER} / \mathrm{BER}, \mathcal{C})\right|_{\mathrm{dB}}-10 \log _{10}\left(2^{2 \cdot R}-1\right)
$$

where $\left.\operatorname{SNR}(\mathrm{FER} / \mathrm{BER}, \mathcal{C})\right|_{\mathrm{dB}}$ denotes the SNR in $\mathrm{dB}$ that is necessary to achieve the desired FER/BER with code $\mathcal{C}$ and

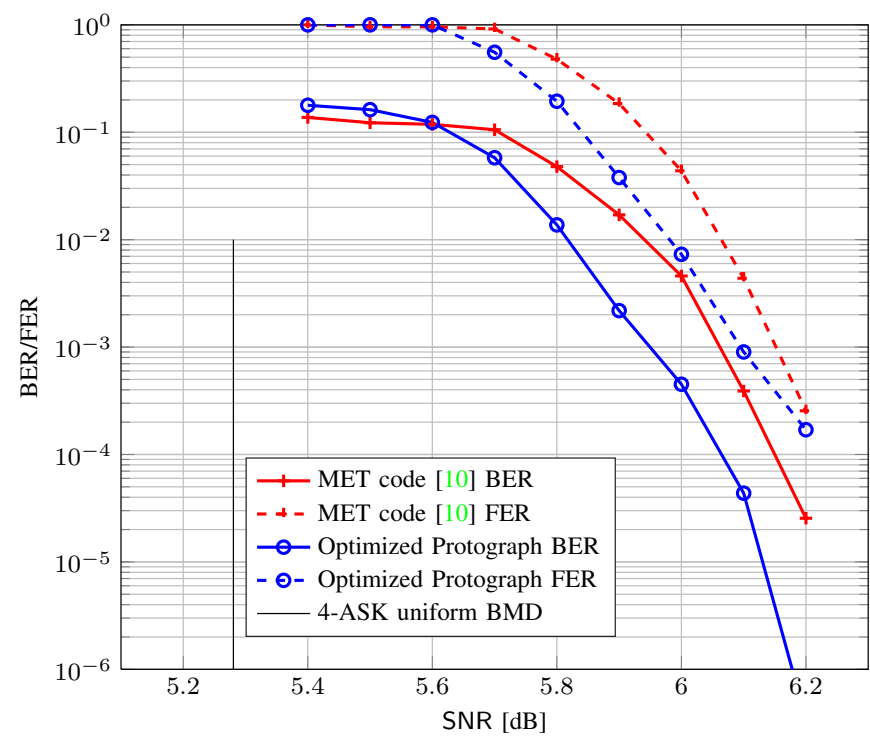

Figure 7. 4-ASK, uniform inputs: Performance of a rate $1 / 2$ optimized protograph code compared to the rate $1 / 2$ MET code in [10].

the rate $R$ equals the transmission rate $\mathbb{H}(\mathbf{B})-1$ at this specific SNR. We use the same input distributions for our optimized codes and for the DVB-S2 codes.

As for the uniform case, the optimized protograph basematrices have been lifted yielding quasi-cyclic parity-check matrices of blocklengths $n=64800.100$ decoding iterations have been performed. The simulation results are depicted in Fig. 9 and 10: At a target FER of $10^{-3}$, the rate $2 / 3$ code has a gap of $0.63 \mathrm{~dB}$ to the AWGN capacity $\frac{1}{2} \log _{2}(1+\mathrm{SNR})$ at a spectral efficiency of $1.38 \mathrm{bits} / \mathrm{channel}$ use. For the $5 / 6$ 


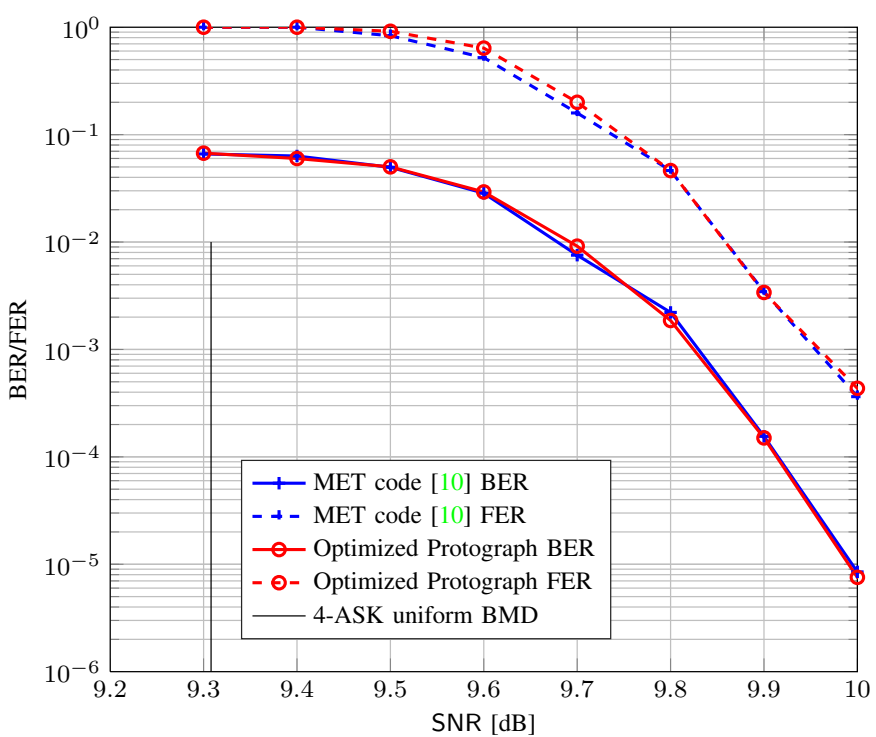

Figure 8. 4-ASK, uniform inputs: Performance of a rate 3/4 optimized protograph code compared to the rate 3/4 MET code in [10].

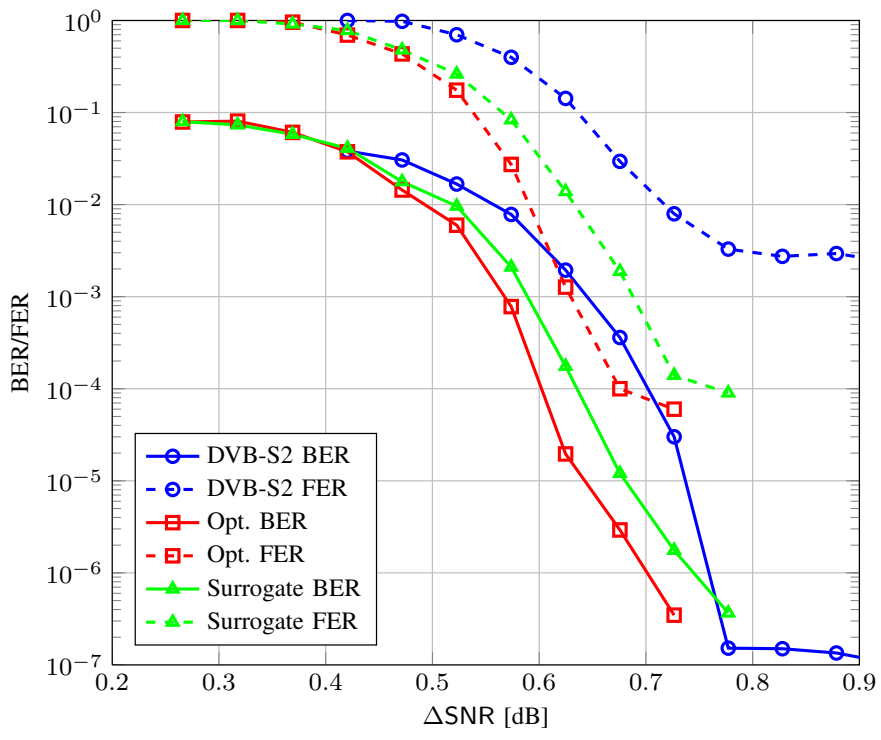

Figure 9. 8-ASK, shaped inputs: Performance comparison of optimized rate $2 / 3$ code and bit-mapping optimized DVB-S2 code. The horizontal axis denotes the gap to AWGN capacity as explained in (25).

rate code, we observe a gap of $0.69 \mathrm{~dB}$ compared to AWGN capacity at a target FER of $10^{-3}$ and a spectral efficiency of 4.25 bits/channel use. Hence, the bit-mapping optimized DVBS2 codes are outperformed by $0.1 \mathrm{~dB}(2 / 3$ code) and $0.25 \mathrm{~dB}$ (5/6 code).

\section{CONCLUSION}

We proposed a protograph-based LDPC code design approach for bandwidth-efficient coded modulation that is suitable both for uniform and shaped inputs. The different bit-

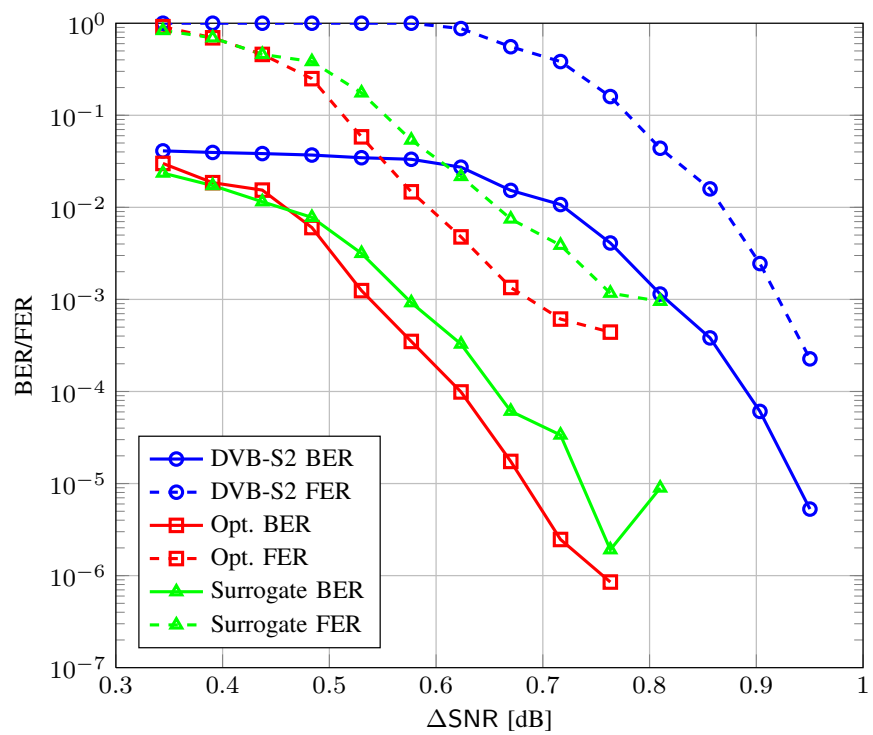

Figure 10. 64-ASK, shaped inputs: Performance comparison of rate 5/6 code and bit-mapping optimized DVB-S2 code. The horizontal axis denotes the gap to AWGN capacity as explained in (25).

channels are replaced by biAWGN surrogates so that PEXIT and differential evolution give ensembles with good decoding thresholds. The performance of the new codes for uniform inputs are as good as the best codes in literature. For shaped inputs, the new codes operate within $0.7 \mathrm{~dB}$ to $\frac{1}{2} \log _{2}(1+$ SNR). Future research should investigate the influence of the surrogates on the code performance by employing a full-fledged density evolution for protographs. Furthermore, precoded protographs should be considered to improve the threshold without increasing the variable node degrees.

\section{REFERENCES}

[1] E. Zehavi, "8-PSK trellis codes for a Rayleigh channel," IEEE Trans. Commun., vol. 40, no. 5, pp. 873-884, May 1992.

[2] G. Caire, G. Taricco, and E. Biglieri, "Bit-interleaved coded modulation," IEEE Trans. Inf. Theory, vol. 44, no. 3, pp. 927-946, 1998.

[3] A. Martinez, A. Guillen i Fabregas, G. Caire, and F. Willems, "Bitinterleaved coded modulation revisited: A mismatched decoding perspective," IEEE Trans. Inf. Theory, vol. 55, no. 6, pp. 2756-2765, June 2009.

[4] A. Guillén i Fàbregas and A. Martinez, "Bit-interleaved coded modulation with shaping," in IEEE Inf. Theory Workshop (ITW), 2010.

[5] G. Böcherer, "Probabilistic signal shaping for bit-metric decoding," in Proc. IEEE Int. Symp. Inf. Theory (ISIT), June 2014, pp. 431-435.

[6] _ , "Achievable rates for shaped bit-metric decoding," arXiv preprint, 2015. [Online]. Available: http://arxiv.org/abs/1410.8075

[7] Y. Li and W. Ryan, "Bit-reliability mapping in LDPC-coded modulation systems," IEEE Commun. Lett., vol. 9, no. 1, pp. 1-3, Jan 2005.

[8] J. Lei and W. Gao, "Matching graph connectivity of LDPC codes to high-order modulation by bit interleaving," in Proc. Allerton Conf. Commun., Contr., Comput., Sept 2008, pp. 1059-1064.

[9] C. Häger, A. Graell i Amat, A. Alvarado, F. Brännström, and E. Agrell, "Optimized bit mappings for spatially coupled LDPC codes over parallel binary erasure channels," in Proc. IEEE Int. Conf. Commun. (ICC), Jun. 2014, pp. 2064-2069.

[10] L. Zhang and F. Kschischang, "Multi-edge-type low-density paritycheck codes for bandwidth-efficient modulation," IEEE Trans. Commun., vol. 61, no. 1, pp. 43-52, January 2013. 
[11] T. Richardson and R. Urbanke, "Multi-edge type LDPC codes," Workshop honoring Prof. Bob McEliece on his 60th birthday, California Institute of Technology, Pasadena, California, pp. 24-25, 2002.

[12] S. ten Brink, "Convergence of iterative decoding," Electron. Lett., vol. 35, no. 10, pp. 806-808, 1999.

[13] J. Thorpe, "Low-density parity-check (LDPC) codes constructed from protographs," IPN progress report, vol. 42, no. 154, pp. 42-154, 2003

[14] D. Divsalar and C. Jones, "Protograph based low error floor LDPC coded modulation," in IEEE Mil. Commun. Conf. (MILCOM), Oct 2005, pp. 378-385 Vol. 1.

[15] A. Marinoni, P. Savazzi, and R. Wesel, "Protograph-based q-ary LDPC codes for higher-order modulation," in Proc. Int. Symp. Turbo Codes and Iterative Inf. Process. (ISTC), Sep. 2010, pp. 68-72.

[16] G. Liva and M. Chiani, "Protograph LDPC code design based on EXIT analysis," in IEEE Global Telecommun. Conf. (GLOBECOM), 2007, pp. 3250-3254.

[17] F. Gray, "Pulse code communication," Mar. 17 1953, US Patent $2,632,058$.

[18] T. Richardson and R. Urbanke, Modern Coding Theory. Cambridge Univ. Press, 2008.

[19] A. Guillén i Fàbregas, A. Martinez, and G. Caire, "Bit-interleaved coded modulation," Found. Trends Comm. Inf. Theory, vol. 5, no. 1-2, pp. 1$153,2008$.

[20] P. Schulte and G. Böcherer, "Constant composition distribution matching," arXiv preprint, 2015. [Online]. Available: http://arxiv.org/ abs/1503.05133

[21] G. Böcherer, P. Schulte, and F. Steiner, "Bandwidth efficient and ratematched low-density parity-check coded modulation," arXiv preprint 2015. [Online]. Available: http://arxiv.org/abs/1502.02733

[22] G. Böcherer, "Capacity-achieving probabilistic shaping for noisy and noiseless channels," Ph.D. dissertation, RWTH Aachen University, 2012.

[23] P. Pulini, G. Liva, and M. Chiani, "Unequal diversity LDPC codes for relay channels," IEEE Trans. Wireless Commun., vol. 12, no. 11, pp. 5646-5655, Nov. 2013.

[24] T. J. Richardson, M. A. Shokrollahi, and R. L. Urbanke, "Design of capacity-approaching irregular low-density parity-check codes," IEEE Trans. Inf. Theory, vol. 47, no. 2, pp. 619-637, 2001.

[25] R. Storn and K. Price, "Differential evolution-a simple and efficient heuristic for global optimization over continuous spaces," Journal of global optimization, vol. 11, no. 4, pp. 341-359, 1997. 